\title{
Energy Shaping Control for a Class of Underactuated Euler-Lagrange Systems *
}

\author{
Alin Albu-Schäffer* Christian Ott Florian Petit \\ * Institute of Robotics and Mechatronics, German Aerospace Center \\ (DLR), Germany \\ (e-mail: Alin.Albu-Schaeffer@dlr.de).
}

\begin{abstract}
The paper presents a new energy shaping control design for a class of underactuated Euler-Lagrange systems. Flexible joint robots, Series Elastic Actuators, and Variable Impedance Actuated Robots Albu-Schäffer et al. [2008] belong for example to this class. First, classical PD control with feed-forward compensation is revisited and a novel, straight-forward and general formulation for the stability analysis is given. Lower bound conditions for the gains of this controller motivate the introduction of the new approach, which generalizes results from Albu-Schäffer et al. [2007], Ch. Ott et al. [2008]. For shaping the potential energy, feedback variables based on the collocated states are introduced, which are statically equivalent to the noncollocated state variables. In this way the passivity is ensured while exactly satisfying steady state requirements formulated in terms of the noncollocated states (such as desired equilibrium configuration and desired stiffness). Using the passivity property, a Lyapunov based analysis can be easily carried out for arbitrarily low feedback gains. The controller is augmented by noncollocated feedback to shape the kinetic energy. Experimental results for a Variable Stiffness Robot Grebenstein et al. [2011] validate the proposed controller.
\end{abstract}

Keywords: Flexible joints, underactuated systems, variable impedance actuation, impedance control.

\section{INTRODUCTION}

Underactuated Euler-Lagrange systems frequently arise in technical context when elasticity plays a significant role. In such a case rigid body models need to be extended by taking elastic properties into account. In the last decade compliance, even nonlinear compliance, has been recognized as a potential way to improve robustness and peak performance of robots, inspired by the archetype of biological musculo-skeletal systems Albu-Schäffer et al. [2008]. However, stabilizing underactuated Euler-Lagrange systems is a challenging task in practice due to the fact that highly accurate models and/or high derivatives of states are mostly required. Energy shaping based control has in this context substantial advantages in terms of robustness with respect to model uncertainties. However, while energy shaping control for fully actuated systems is well established providing constructive design methods Takegaki and Arimoto [1981], Ortega and M.Spong [1989], Tomei [1991], Blankenstein et al. [nn], Ortega and M.Spong [2002], Ortega et al. [2002], van der Schaft [2002], Siciliano et al. [2009], for the case of underactuated E$\mathrm{L}$ systems no constructive solution is available to our knowledge so far. In Blankenstein et al. [nn], Ortega and M.Spong [2002], Ortega et al. [2002] a system of partial differential equations (PDE) has to be solved in order to find the energy function and the controller, what in general is a quite difficult task.

\footnotetext{
* This paper has been partly funded by the European commissions Seventh Framework Program as part of the project SAPHARI (Grant Agreement no. 287513) and THE (Grant agreement no. 248587).
}

The paper addresses underactuated systems which can be stabilized by shaping only the potential energy. For these systems, a one-to-one relationship is given between the collocated and the noncollocated state variables in static configurations. We would call these systems "fully potentially coupled underactuated systems", in contrast to "inertially coupled underactuated systems", for which such a one-to-one static relationship does not hold ${ }^{1}$. The considered systems can be in principle stabilized by a feedforward compensation at the desired configuration and a PD-type controller. A general, straight forward formulation for their stability analysis is introduced for the first time in this paper. However, this simple approach requires lower bounds on the controller gains which might be restrictive in practice and suffers from inaccuracy of the feed-forward compensation for larger displacements from equilibrium. The main idea for the new controller is to design the shaping of the potential energy by introducing a new control variable, which is a function of the collocated state variables only, but is equal to the noncollocated state variables in any static configuration. A (numerical) solution to an algebraic equation has to be found in order to calculate these variables. A collocated and passive controller can be designed this way, while exactly fulfilling the steady state requirements for the system. If the system satisfies some specified conditions, there is a straight-forward way to define the controller and the corresponding energy function. The paper is origi-

\footnotetext{
1 Note that the term "fully potentially coupled" does not exclude the additional presence of inertial couplings between the collocated and non-collocated states.
} 
nating from developments in Cartesian impedance control of flexible joint robots Albu-Schäffer et al. [2005, 2007], Ch. Ott et al. [2008] and contains a generalization of the approach presented therein, allowing to treat for example fully coupled, nonlinear stiffness potentials. Revisiting this topic after first addressing it in the context of impedance control Albu-Schäffer et al. [2005] is motivated by the revival of interest in this type of underactuated systems due to recent intensive research on robots with Variable Impedance Actuation (VIA) Morita et al. [1999], Bicchi and Tonietti [2004], Migliore et al. [2005], Palli et al. [2007], Vanderborght et al. [2006], Koganezawa [2005], English and Russell [1999], Morita and Sugano [1997], Hurst and Rizzi [2008], Wolf and Hirzinger [2008], Albu-Schäffer et al. [2008], Wolf et al. [2011], Petit et al. [2010], web site [2011]. Due to the very large, nonlinear compliance of VIA systems, the proposed method has special relevance in this context.

A controller based solely on the collocated variables may, however, have a limited control performance. In order to increase performance, an inner control loop is proposed for the considered class of systems. Its effect is interpreted as the shaping of the kinetic energy of the actuators. The controller itself is not passive any more, but it will be shown that the controlled actuator dynamics in combination with the torque feedback controller is passive. Based on these passivity properties, the asymptotic stability can be shown by invoking La Salle's invariance principle.

The derived conditions are discussed and some simple examples are given. Based on the presented method, a controller for VIA robots is derived as an example. Finally experimental results with the VSA-based DLR hand-armsystem Grebenstein et al. [2011] validate the method.

\section{PROBLEM STATEMENT}

Consider an Euler-Lagrange system with damping, satisfying:

$$
\frac{d}{d t}\left(\frac{\partial L}{\partial \dot{\boldsymbol{x}}}\right)-\frac{\partial L}{\partial \boldsymbol{x}}=\boldsymbol{\tau}_{m}-\boldsymbol{D} \dot{\boldsymbol{x}} .
$$

The Lagrangian $L(\boldsymbol{x}, \dot{\boldsymbol{x}})=T(\boldsymbol{x}, \dot{\boldsymbol{x}})-U(\boldsymbol{x})$ is the difference of the kinetic energy ${ }^{2} T(\boldsymbol{x}, \dot{\boldsymbol{x}})$ and the potential energy $U(\boldsymbol{x})$. Conditions for the potential energy $U(\boldsymbol{x})$ will be formulated in Sec. 2.1 to closer specify the class of systems for which the proposed control approach is valid. $\boldsymbol{x} \in \mathbb{R}^{n}$ is the vector of generalized coordinates, $\boldsymbol{\tau}_{m} \in \mathbb{R}^{n}$ the vector of generalized control input forces. Furthermore, the system contains a dissipative friction force specified by the positive definite damping matrix ${ }^{3} \boldsymbol{D} \in \mathbb{R}^{n \times n}$. In the case of an underactuated system with $k$ independent actuators, the control input has the form

$$
\boldsymbol{\tau}_{m}=\left(\begin{array}{l}
\boldsymbol{u} \\
\mathbf{0}
\end{array}\right), \quad \text { with } \quad \boldsymbol{u} \in \mathbb{R}^{k}, k \leq n .
$$

The general task we would like to address is the control of $k$ independent output variables given by

$$
\boldsymbol{y}=\boldsymbol{h}(\boldsymbol{x})
$$

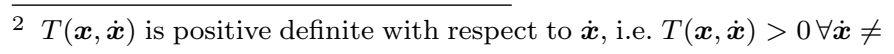
0 .

3 The results can be easily extended to the case of positive semidefinite damping matrices using results from dissipative systems analysis van der Schaft [2002]. to desired constant values $\boldsymbol{y}_{d} \in \mathbb{R}^{k}$. Accordingly, the state vector can be then partitioned into $\boldsymbol{x}=(\boldsymbol{\theta}, \boldsymbol{q})$, with $\boldsymbol{\theta} \in \mathbb{R}^{k}$ being the directly actuated states corresponding to the input $\boldsymbol{u}$ and $\boldsymbol{q} \in \mathbb{R}^{n-k}$ being the un-actuated states. A simplified version of this problem will be formulated next, in order to present the main idea in a compact form in this paper. The extension to the general case specified above, required to treat for example the general VIA model introduced in Albu-Schäffer et al. [2010] is currently in preparation.

\subsection{Simplified problem $(n=2 k)$}

Let us first consider a system with $n=2 k$. In this case $\boldsymbol{\theta}, \boldsymbol{q} \in \mathbb{R}^{k}$. Furthermore, consider the mapping $\boldsymbol{h}(\boldsymbol{x})$ to be chosen as such that $\boldsymbol{y}=\boldsymbol{q}$. The task is therefore to control $\boldsymbol{q}$ to a desired constant value $\boldsymbol{q}_{d}$.

The equation of the equilibrium for (1), obtained by setting the state derivatives $\dot{\boldsymbol{x}}$ (and therefore also the kinetic energy $T(\boldsymbol{x}, \dot{\boldsymbol{x}}))$ to zero, is:

$$
\frac{\partial U(\boldsymbol{x})}{\partial \boldsymbol{x}}=\boldsymbol{\tau}_{m}
$$

Using the partitioning into actuated and non-actuated states, this leads to

$$
\begin{aligned}
& \boldsymbol{f}_{\theta}(\boldsymbol{\theta}, \boldsymbol{q}):=\frac{\partial U(\boldsymbol{\theta}, \boldsymbol{q})}{\partial \boldsymbol{\theta}}=\boldsymbol{u} \\
& \boldsymbol{f}_{\boldsymbol{q}}(\boldsymbol{\theta}, \boldsymbol{q}):=\frac{\partial U(\boldsymbol{\theta}, \boldsymbol{q})}{\partial \boldsymbol{q}}=\mathbf{0} .
\end{aligned}
$$

Consider a desired equilibrium point $\boldsymbol{x}_{d}=\left(\boldsymbol{\theta}_{d}, \boldsymbol{q}_{d}\right)$ which satisfies (6). Now let us assume that the following second order partial derivatives are bounded, i.e. there exists constants $\alpha_{1}, \alpha_{2}, \alpha_{3}, \alpha_{4}>0$ such that the inequalities

$$
\begin{gathered}
\alpha_{2}\|\boldsymbol{q}\|^{2}>\boldsymbol{q}^{T} \frac{\partial^{2} U(\boldsymbol{\theta}, \boldsymbol{q})}{\partial \boldsymbol{q}^{2}} \boldsymbol{q}>\alpha_{1}\|\boldsymbol{q}\|^{2} \\
\alpha_{3}>\left\|\frac{\partial^{2} U(\boldsymbol{\theta}, \boldsymbol{q})}{\partial \boldsymbol{q} \partial \boldsymbol{\theta}}\right\|>\alpha_{4}
\end{gathered}
$$

hold ${ }^{4} \forall \boldsymbol{x} \in \mathbb{R}^{n}$. These are the central assumptions on the structure of the Lagrangian, which define the class of systems for which the control approach can be applied. Sec. 5 is dedicated to the interpretation and discussion of these conditions.

Additionally, for Sec. 3 only, we assume that $\left\|\frac{\partial^{2} U(\boldsymbol{\theta}, \boldsymbol{q})}{\partial \boldsymbol{\theta}^{2}}\right\|$ is bounded from below, i.e. there exists $\alpha_{5} \in \mathbb{R}$ such that

$$
\boldsymbol{\theta}^{T} \frac{\partial^{2} U(\boldsymbol{\theta}, \boldsymbol{q})}{\partial \boldsymbol{\theta}^{2}} \boldsymbol{\theta}>\alpha_{5}\|\boldsymbol{\theta}\|^{2} .
$$

\section{THE CLASSICAL APPROACH: PD CONTROL WITH FEED-FORWARD COMPENSATION BASED ON DESIRED POSITION}

A very simple (but restrictive) approach to the control problem stated above uses only the desired and measured values of the collocated states for the controller. A typical controller structure is as follows:

$$
\boldsymbol{u}=\boldsymbol{f}_{\theta}\left(\boldsymbol{\theta}_{d}, \boldsymbol{q}_{d}\right)-\boldsymbol{K}_{p 1} \tilde{\boldsymbol{\theta}}-\boldsymbol{K}_{d} \dot{\boldsymbol{\theta}},
$$

4 The usual Euclidean norm for vectors and the corresponding induced matrix norm is assumed throughout the paper. 
with $\tilde{\boldsymbol{\theta}}=\boldsymbol{\theta}-\boldsymbol{\theta}_{d}$. This is basically a PD controller with feed-forward compensation, as suggested by the classical linearization approach. However, under the conditions (7), (8), (9) global convergence can be shown if $\left\|\boldsymbol{K}_{p 1}\right\|$ is sufficiently large. While such controllers have been often proposed for particular robotic systems Takegaki and Arimoto [1981], Tomei [1991], Albu-Schäffer and Hirzinger [2001], we formulate the approach here for the quite general underactuated system defined above and provide a novel, straight-forward and compact approach for choosing a Lyapunov function. Therefore, although mainly introduced for readability and motivation purposes, this section has in our view a substantial value by itself.

A Lyapunov function for the closed loop system (1), (2), (10) is given by

$V_{1}(\boldsymbol{x}, \dot{\boldsymbol{x}})=T(\boldsymbol{x}, \dot{\boldsymbol{x}})+V_{P 1}(\boldsymbol{x})=T(\boldsymbol{x}, \dot{\boldsymbol{x}})+U(\boldsymbol{x})+V_{C 1}(\boldsymbol{x})$,

with the total potential energy $V_{P 1}(\boldsymbol{x})$ being the sum of the plant potential energy $U(\boldsymbol{x})$ and the controller energy

$$
V_{C 1}(\boldsymbol{x})=-U\left(\boldsymbol{x}_{d}\right)-\frac{\partial U^{T}}{\partial \boldsymbol{x}}\left(\boldsymbol{x}_{d}\right)\left(\boldsymbol{x}-\boldsymbol{x}_{d}\right)+\frac{1}{2} \tilde{\boldsymbol{\theta}}^{T} \boldsymbol{K}_{p 1} \tilde{\boldsymbol{\theta}}
$$

With the notation

$$
U_{P 1}(\boldsymbol{x})=U(\boldsymbol{x})+\frac{1}{2} \boldsymbol{\theta}^{T} \boldsymbol{K}_{p 1} \boldsymbol{\theta}
$$

the total potential energy takes the very simple form

$$
V_{P 1}(\boldsymbol{x})=U_{P 1}(\boldsymbol{x})-U_{P 1}\left(\boldsymbol{x}_{d}\right)-\frac{\partial U_{P 1}^{T}}{\partial \boldsymbol{x}}\left(\boldsymbol{x}_{d}\right)\left(\boldsymbol{x}-\boldsymbol{x}_{d}\right) .
$$

It can easily be verified that $V_{P 1}\left(\boldsymbol{x}_{d}\right)=0$ and also that $\boldsymbol{x}_{d}$ is an extremal point of $V_{P 1}(\boldsymbol{x})$ since $\frac{\partial V_{P 1}}{\partial \boldsymbol{x}}\left(\boldsymbol{x}_{d}\right)=\mathbf{0}$. It follows that $V_{P 1}(\boldsymbol{x})$ is positive definite if its Hessian ${ }^{5}$, given by

$$
H_{P 1}(\boldsymbol{x})=\left[\begin{array}{cc}
\frac{\partial^{2} U(\boldsymbol{\theta}, \boldsymbol{q})}{\partial \boldsymbol{q}^{2}} & \frac{\partial^{2} U(\boldsymbol{\theta}, \boldsymbol{q})}{\partial \boldsymbol{q} \partial \boldsymbol{\theta}} \\
\frac{\partial^{2} U(\boldsymbol{\theta}, \boldsymbol{q})}{\partial \boldsymbol{\theta} \partial \boldsymbol{q}} & \frac{\partial^{2} U(\boldsymbol{\theta}, \boldsymbol{q})}{\partial \boldsymbol{\theta}^{2}}+\boldsymbol{K}_{p 1}
\end{array}\right]
$$

is positive definite. In this case $\boldsymbol{x}_{d}$ is the only extremal point. The equilibrium points of the system, given by

$$
\begin{aligned}
& \boldsymbol{f}_{\theta}(\boldsymbol{\theta}, \boldsymbol{q})=\boldsymbol{f}_{\theta}\left(\boldsymbol{\theta}_{d}, \boldsymbol{q}_{d}\right)-\boldsymbol{K}_{p 1} \tilde{\boldsymbol{\theta}} \\
& \boldsymbol{f}_{\boldsymbol{q}}(\boldsymbol{\theta}, \boldsymbol{q})=\mathbf{0}
\end{aligned}
$$

correspond to the extrema of $V_{P 1}(\boldsymbol{x})$, implying that $\boldsymbol{x}_{d}$ is in this case the only equilibrium point.

It can be easily seen that under the conditions $(7),(8),(9)$ it is always possible to choose the gain matrix $\boldsymbol{K}_{p 1}$ high enough, such that $H_{P 1}(\boldsymbol{x})$, and consequently $V_{1}(\boldsymbol{x}, \dot{\boldsymbol{x}})=$ $T(\boldsymbol{x}, \dot{\boldsymbol{x}})+V_{P 1}(\boldsymbol{x})$ are positive definite ${ }^{6}$. The plant is obviously passive with respect to $\boldsymbol{u}, \boldsymbol{\theta}$ while the controller is passive w.r.t. $\boldsymbol{\theta},-\boldsymbol{u}$ since

$$
\begin{aligned}
\dot{V}_{C 1}(\boldsymbol{x}) & =-\frac{\partial U^{T}}{\partial \boldsymbol{x}}\left(\boldsymbol{x}_{d}\right) \dot{\boldsymbol{x}}+\tilde{\boldsymbol{\theta}}^{T} \boldsymbol{K}_{p 1} \dot{\boldsymbol{\theta}} \\
& =\left(-\frac{\partial U^{T}}{\partial \boldsymbol{\theta}}\left(\boldsymbol{x}_{d}\right)+\tilde{\boldsymbol{\theta}}^{T} \boldsymbol{K}_{p 1}\right) \dot{\boldsymbol{\theta}} \\
& =-\boldsymbol{u}^{T} \dot{\boldsymbol{\theta}}-\dot{\boldsymbol{\theta}}^{T} \boldsymbol{K}_{d} \dot{\boldsymbol{\theta}}
\end{aligned}
$$

\footnotetext{
5 which is identical to the Hessian of $U_{P 1}(\boldsymbol{x})$

6 Actually, it is quite straight-forward to see that the positive definiteness condition is fulfilled if $\left\|\boldsymbol{K}_{p 1}+\frac{\partial^{2} U(\boldsymbol{\theta}, \boldsymbol{q})}{\partial \boldsymbol{\theta}^{2}}\right\|>\alpha_{3}^{2} / \alpha_{1}$ or, more conservatively, $\left\|\boldsymbol{K}_{p 1}\right\|>\alpha_{3}^{2} / \alpha_{1}+\left|\alpha_{5}\right|$.
}

where (10) and the fact that $\boldsymbol{f}_{q}\left(\boldsymbol{x}_{d}\right)=\mathbf{0}$ have been used. Therefore we have

$$
\dot{V}_{1}(\boldsymbol{x}, \dot{\boldsymbol{x}})=-\dot{\boldsymbol{x}}^{T} \boldsymbol{D} \dot{\boldsymbol{x}}-\dot{\boldsymbol{\theta}}^{T} \boldsymbol{K}_{d} \dot{\boldsymbol{\theta}}
$$

from which we can conclude stability. Moreover, asymptotic stability of the closed loop system can be shown using La Salle's theorem.

Remark 1: The equilibrium conditions (16), (17) can be written due to $\boldsymbol{f}_{q}\left(\boldsymbol{x}_{d}\right)=\mathbf{0}$ as

$$
\begin{aligned}
\boldsymbol{f}_{\theta}(\boldsymbol{\theta}, \boldsymbol{q})+\boldsymbol{K}_{p 1} \boldsymbol{\theta} & =\boldsymbol{f}_{\theta}\left(\boldsymbol{\theta}_{d}, \boldsymbol{q}_{d}\right)+\boldsymbol{K}_{p 1} \boldsymbol{\theta}_{d} \\
\boldsymbol{f}_{\boldsymbol{q}}(\boldsymbol{\theta}, \boldsymbol{q}) & =\boldsymbol{f}_{\boldsymbol{q}}\left(\boldsymbol{\theta}_{d}, \boldsymbol{q}_{d},\right)
\end{aligned}
$$

or

$$
\boldsymbol{f}_{t o t}(\boldsymbol{x})-\boldsymbol{f}_{t o t}\left(\boldsymbol{x}_{d}\right)=\mathbf{0}
$$

with $\boldsymbol{f}_{\text {tot }}(\boldsymbol{x})=\left[\begin{array}{c}\boldsymbol{f}_{\theta}(\boldsymbol{\theta}, \boldsymbol{q})+\boldsymbol{K}_{p 1} \boldsymbol{\theta} \\ \boldsymbol{f}_{\boldsymbol{q}}(\boldsymbol{\theta}, \boldsymbol{q})\end{array}\right]$. Note that (14) can be then recognized as

$$
V_{P 1}(\boldsymbol{x})=\int_{x_{d}}^{x}\left(\boldsymbol{f}_{\text {tot }}(\boldsymbol{x})-\boldsymbol{f}_{\text {tot }}\left(\boldsymbol{x}_{d}\right)\right) \mathrm{d} \boldsymbol{x} .
$$

Remark 2: The controller presented in this section is a high gain controller. In practice, the lower bounds for $\boldsymbol{K}_{p 1}$ may be quite restrictive. In some particular cases, as for example in impedance or stiffness control Hogan [1985], Albu-Schäffer et al. [2007], one may want to implement controller gains arbitrarily close to zero. This is obviously not possible using this approach. In contrast, the method introduced in the next section does not impose lower bounds on the controller gain matrix $\boldsymbol{K}_{p 1}$, allowing it to be any positive definite matrix. Moreover, the feed-forward compensation will not be done based on desired values, but on measured ones, providing higher performance for large displacements from the desired equilibrium.

\section{PASSIVE, LINK SIDE EQUIVALENT CONTROLLER}

Controller (10) contains a nonlinear compensation at the equilibrium point and a collocated state PD-type feedback. Obviously, the feed-forward compensation is inaccurate for displacements from the equilibrium. The imposed conditions, however, ensure that the restoring proportional term grows faster than this error, if the proportional gain is high enough. In order to permit arbitrarily small proportional gains we need to provide a more precise feed-forward compensation based on current values. To obtain a passive controller, a collocated feedback, using only directly actuated states $\boldsymbol{\theta}$ and $\dot{\boldsymbol{\theta}}$ is needed. In this section we develop a controller fulfilling these requirements.

Due to property (7) equation (6) has exactly one solution for $\boldsymbol{q}$ for every value of $\boldsymbol{\theta}$ (see Appendix). This implicitly defined function will be denoted by $\overline{\boldsymbol{q}}$ :

$$
\exists \overline{\boldsymbol{q}}: \mathbb{R}^{k} \rightarrow \mathbb{R}^{k} \text { such that } \boldsymbol{f}_{q}(\boldsymbol{\theta}, \overline{\boldsymbol{q}}(\boldsymbol{\theta}))=\mathbf{0}, \forall \boldsymbol{\theta} \in \mathbb{R}^{k},
$$

Property (24) results from the fact that $U(\boldsymbol{x})$ is positive definite with respect to $\boldsymbol{q}$ and has exactly one extremum for each $\boldsymbol{\theta}$ therein.

The matrix of partial derivatives of $\overline{\boldsymbol{q}}$ satisfies then

$$
\boldsymbol{J}_{\bar{q}}(\boldsymbol{\theta})=\frac{\partial \overline{\boldsymbol{q}}(\boldsymbol{\theta})}{\partial \boldsymbol{\theta}}=-\left(\frac{\partial^{2} U(\boldsymbol{\theta}, \overline{\boldsymbol{q}})}{\partial \boldsymbol{q}^{2}}\right)^{-1} \frac{\partial^{2} U(\boldsymbol{\theta}, \overline{\boldsymbol{q}})}{\partial \boldsymbol{q} \partial \boldsymbol{\theta}} .
$$

This follows directly by differentiating $\boldsymbol{f}(\boldsymbol{\theta}, \overline{\boldsymbol{q}})$ with respect to $\boldsymbol{\theta}$. As shown in the Appendix, if the properties (7), 
(8) are satisfied then $\boldsymbol{J}_{\bar{q}}(\boldsymbol{\theta})$ is nonsingular and $\overline{\boldsymbol{q}}(\boldsymbol{\theta})$ is a diffeomorphism.

Remark: In most cases, it will not be possible to solve equation (6) analytically. However, it is ensured that the equation has exactly one solution and that the problem of numerically finding this solution has only one minimum due to its convex nature. It is therefore reasonable to assume that the equation can be solved with existing numerical methods up to a sufficient accuracy in short time. This implies mainly some requirements on the available computation power. For a detailed discussion of this topic and its implications see sec. 6 . It will be thus assumed in the following that $\overline{\boldsymbol{q}}(\boldsymbol{\theta})$ is available for the further controller design.

The main idea in the controller design is to use the new variable $\overline{\boldsymbol{q}}(\boldsymbol{\theta})$ for the controller feedback instead of $\boldsymbol{q}$ or $\boldsymbol{\theta}$ in order to stabilize the system around $\boldsymbol{x}_{d}$. This variable was chosen such that it will be equal to $\boldsymbol{q}$ in any static situation, i.e.

$$
\forall \boldsymbol{\theta} \in \mathbb{R}^{k}: \quad \dot{\boldsymbol{x}}=\mathbf{0} \Rightarrow \boldsymbol{q}=\overline{\boldsymbol{q}}(\boldsymbol{\theta}),
$$

such that one can construct a collocated controller, which is statically equivalent to a noncollocated one based on $\boldsymbol{q}$. Using (5), the following control input can be defined:

$$
\boldsymbol{u}=\boldsymbol{f}_{\theta}(\boldsymbol{\theta}, \overline{\boldsymbol{q}}(\boldsymbol{\theta}))-\boldsymbol{J}_{\bar{q}}^{T}(\boldsymbol{\theta}) \boldsymbol{K}_{p} \boldsymbol{e}(\boldsymbol{\theta})-\boldsymbol{K}_{d} \dot{\boldsymbol{\theta}},
$$

with $\left(\boldsymbol{e}(\boldsymbol{\theta})=\overline{\boldsymbol{q}}(\boldsymbol{\theta})-\boldsymbol{q}_{d}\right) . \boldsymbol{K}_{p}$ is a constant, positive definite, symmetric gain matrix and $\boldsymbol{K}_{d}$ is a (possibly) state dependent, positive definite damping matrix.

\subsection{Lyapunov function}

Consider the following Lyapunov function candidate for the closed loop system:

$$
V(\boldsymbol{x}, \dot{\boldsymbol{x}})=T(\boldsymbol{x}, \dot{\boldsymbol{x}})+U(\boldsymbol{\theta}, \boldsymbol{q})+V_{C}(\boldsymbol{\theta}, \overline{\boldsymbol{q}})
$$

with

$$
V_{C}(\boldsymbol{\theta}, \overline{\boldsymbol{q}})=-U(\boldsymbol{\theta}, \overline{\boldsymbol{q}})+\frac{1}{2} \boldsymbol{e}(\boldsymbol{\theta})^{T} \boldsymbol{K}_{p} \boldsymbol{e}(\boldsymbol{\theta}) .
$$

Remark: Note that

$$
\frac{\partial U(\boldsymbol{\theta}, \overline{\boldsymbol{q}}(\boldsymbol{\theta}))}{\partial \boldsymbol{\theta}}=\boldsymbol{f}_{\theta}(\boldsymbol{\theta}, \overline{\boldsymbol{q}}(\boldsymbol{\theta})),
$$

since $\boldsymbol{f}_{q}(\boldsymbol{\theta}, \overline{\boldsymbol{q}}(\boldsymbol{\theta}))=\mathbf{0}$. Therefore

$$
\begin{aligned}
\dot{V}_{C}(\boldsymbol{\theta}, \overline{\boldsymbol{q}}) & =-\dot{\boldsymbol{\theta}}^{T}\left(\boldsymbol{f}_{\theta}(\boldsymbol{\theta}, \overline{\boldsymbol{q}}(\boldsymbol{\theta}))-\boldsymbol{J}_{\bar{q}}^{T}(\boldsymbol{\theta}) \boldsymbol{K}_{p} \boldsymbol{e}(\boldsymbol{\theta})\right) \\
& =-\boldsymbol{u}^{T} \dot{\boldsymbol{\theta}}-\dot{\boldsymbol{\theta}}^{T} \boldsymbol{K}_{d} \dot{\boldsymbol{\theta}}
\end{aligned}
$$

and thus $V_{C}(\boldsymbol{\theta}, \overline{\boldsymbol{q}})$ is a "candidate energy function" for the controller, which is passive if the potential energy $U(\boldsymbol{x})$ is bounded from below.

For the desired equilibrium configuration defined by $\{\boldsymbol{x}=$ $\left.\boldsymbol{x}_{d}, \dot{\boldsymbol{x}}=\mathbf{0}\right\}$ the required property $V\left(\boldsymbol{x}_{d}, \dot{\boldsymbol{x}}_{d}\right)=0$ is directly verified, due to the fact that in this configuration $\boldsymbol{q}=\overline{\boldsymbol{q}}$ holds.

In order to show that $V(\boldsymbol{x}, \dot{\boldsymbol{x}})$ is positive definite, consider first the difference $\Delta U_{q}(\boldsymbol{\theta}, \boldsymbol{q})=U(\boldsymbol{\theta}, \boldsymbol{q})-U(\boldsymbol{\theta}, \overline{\boldsymbol{q}})$. Showing that $\Delta U_{q}(\boldsymbol{\theta}, \boldsymbol{q})$ is positive for $\boldsymbol{q} \neq \overline{\boldsymbol{q}}$ is equivalent to showing that $U(\boldsymbol{\theta}, \boldsymbol{q})$ has the only extremum at $\boldsymbol{q}=\overline{\boldsymbol{q}}$ for any given $\boldsymbol{\theta}$, which is seen here as a parameter. This follows from the fact that

$$
\frac{\partial U(\boldsymbol{\theta}, \boldsymbol{q})}{\partial \boldsymbol{q}}(\overline{\boldsymbol{q}})=\boldsymbol{f}_{\boldsymbol{q}}(\boldsymbol{\theta}, \overline{\boldsymbol{q}})=\mathbf{0}
$$

and that the Hessian (given by (7)) is positive definite. Furthermore, (7) generally implies

$$
\begin{aligned}
\left|U\left(\boldsymbol{\theta}, \boldsymbol{q}_{1}\right)-U\left(\boldsymbol{\theta}, \boldsymbol{q}_{2}\right)-\left(\boldsymbol{q}_{1}-\boldsymbol{q}_{2}\right)^{T} \boldsymbol{f}_{q}\left(\boldsymbol{\theta}, \boldsymbol{q}_{2}\right)\right| \\
\geq \frac{1}{2} \alpha_{1}\left\|\boldsymbol{q}_{1}-\boldsymbol{q}_{2}\right\|^{2}, \quad \forall \boldsymbol{\theta}, \boldsymbol{q}_{1}, \boldsymbol{q}_{2} \in \mathbb{R}^{k}
\end{aligned}
$$

In particular, for $\boldsymbol{q}_{1}=\boldsymbol{q}, \boldsymbol{q}_{2}=\overline{\boldsymbol{q}}(\boldsymbol{\theta})$ one obtains

$$
\Delta U_{q}(\boldsymbol{\theta}, \boldsymbol{q}) \geq \frac{1}{2}(\boldsymbol{q}-\overline{\boldsymbol{q}})^{T} \alpha_{1}(\boldsymbol{q}-\overline{\boldsymbol{q}})
$$

From (28) it follows that

$$
\begin{aligned}
V(\boldsymbol{x}, \dot{\boldsymbol{x}}) \geq & T(\boldsymbol{x}, \dot{\boldsymbol{x}})+\frac{1}{2}(\boldsymbol{q}-\overline{\boldsymbol{q}})^{T} \alpha_{1}(\boldsymbol{q}-\overline{\boldsymbol{q}}) \\
& +\frac{1}{2} \boldsymbol{e}(\boldsymbol{\theta})^{T} \boldsymbol{K}_{p} \boldsymbol{e}(\boldsymbol{\theta}) \geq 0 .
\end{aligned}
$$

The equality holds only for $\boldsymbol{q}=\overline{\boldsymbol{q}}=\boldsymbol{q}_{d}$, which, considering that $\overline{\boldsymbol{q}}(\boldsymbol{\theta})$ is a diffeomorphism, implies $\boldsymbol{\theta}=\boldsymbol{\theta}_{d}$. It follows that $V(\boldsymbol{x}, \dot{\boldsymbol{x}})=0$ is fulfilled only for $\left\{\boldsymbol{x}=\boldsymbol{x}_{d}, \dot{\boldsymbol{x}}=\mathbf{0}\right\}$.

\subsection{Equilibrium condition}

Using the controller (27), the equilibrium conditions (5),(6) become:

$$
\begin{aligned}
& \boldsymbol{f}_{\theta}(\boldsymbol{\theta}, \boldsymbol{q})=\boldsymbol{f}_{\theta}(\boldsymbol{\theta}, \overline{\boldsymbol{q}}(\boldsymbol{\theta}))-\boldsymbol{J}_{\bar{q}}^{T}(\boldsymbol{\theta}) \boldsymbol{K}_{p} \boldsymbol{e}(\boldsymbol{\theta}) \\
& \boldsymbol{f}_{\boldsymbol{q}}(\boldsymbol{\theta}, \boldsymbol{q})=\mathbf{0}
\end{aligned}
$$

The only solution of (38) is $\boldsymbol{q}=\overline{\boldsymbol{q}}$. By substituting it into (37) it follows that $\overline{\boldsymbol{q}}=\boldsymbol{q}_{d}$, and from the fact that $\overline{\boldsymbol{q}}(\boldsymbol{\theta})$ is a diffeomorphism $\boldsymbol{\theta}=\boldsymbol{\theta}_{d}$ results. It can be therefore concluded that the equilibrium equations have exactly one solution, namely $\boldsymbol{x}=\boldsymbol{x}_{d}$ with $\boldsymbol{x}_{d}=\left(\boldsymbol{\theta}_{d}, \boldsymbol{q}_{d}\right)$.

\subsection{Derivative of the Lyapunov function}

The derivative of the energy function of the plant,

$$
H(\boldsymbol{x}, \dot{\boldsymbol{x}})=T(\boldsymbol{x}, \dot{\boldsymbol{x}})+U(\boldsymbol{x}),
$$

is known to be

$$
\begin{aligned}
\dot{H}(\boldsymbol{x}, \dot{\boldsymbol{x}}) & =-\dot{\boldsymbol{x}}^{T} \boldsymbol{D} \dot{\boldsymbol{x}}+\boldsymbol{\tau}_{m}^{T} \dot{\boldsymbol{x}} \\
& =-\dot{\boldsymbol{x}}^{T} \boldsymbol{D} \dot{\boldsymbol{x}}+\boldsymbol{u}^{T} \dot{\boldsymbol{\theta}}
\end{aligned}
$$

This leads together with (32) to the derivative of the Lyapunov function:

$$
\dot{V}(\boldsymbol{x}, \dot{\boldsymbol{x}})=-\dot{\boldsymbol{x}}^{T} \boldsymbol{D} \dot{\boldsymbol{x}}-\dot{\boldsymbol{\theta}}^{T} \boldsymbol{K}_{d} \dot{\boldsymbol{\theta}}
$$

This function is negative semi-definite. It can be therefore concluded that the system is stable.

\subsection{Global asymptotic stability}

Global asymptotic stability can be shown based on La Salle's invariance theorem. The results can be summarized in the following proposition:

Proposition 1. The system given by (1),(2),(3), together with the controller given by (27) is globally asymptotically stable if the conditions (7),(8) are globally valid.

Proof: As mentioned in sec. 4, (24) holds if (7) holds. In order for $\overline{\boldsymbol{q}}$ to be a global diffeomorphism, it is sufficient that $\boldsymbol{J}_{\bar{q}}(\boldsymbol{\theta})$ is nonsingular. This is fulfilled if (Zeidler [1986], pp.174):

$$
\sup _{\boldsymbol{\theta} \in \mathbb{R}^{k}}\left\|\boldsymbol{J}_{\bar{q}}^{-1}(\boldsymbol{\theta})\right\|<\infty
$$




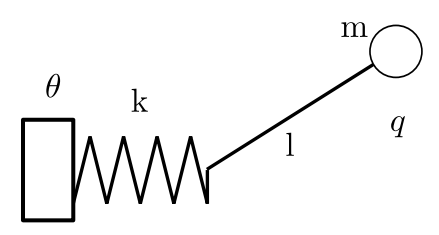

Fig. 1. Pendulum fixed by a spring

In view of $(7),(8)$ this condition is satisfied, since:

$$
\left\|\boldsymbol{J}_{\bar{q}}^{-1}(\boldsymbol{\theta})\right\|<\left\|\left(\frac{\partial^{2} U(\boldsymbol{\theta}, \boldsymbol{q})}{\partial \boldsymbol{q} \partial \boldsymbol{\theta}}\right)^{-1}\right\|\left\|\frac{\partial^{2} U(\boldsymbol{\theta}, \boldsymbol{q})}{\partial \boldsymbol{q}^{2}}\right\|<\alpha_{2} \alpha_{4} .
$$

The Lyapunov function from Sec. 4.1 can be used to show the global asymptotic stability, by additionally noting that from (36) it follows that $V(\boldsymbol{x}, \dot{\boldsymbol{x}}) \rightarrow \infty$ for $\boldsymbol{x} \rightarrow \infty$ or $\dot{\boldsymbol{x}} \rightarrow \infty$ when $\overline{\boldsymbol{q}}(\boldsymbol{\theta})$ is a diffeomorphism. The system state will converge into the largest invariant set for which $\dot{\boldsymbol{x}}=\mathbf{0}$ holds. But there does not exist any trajectory for which $\dot{\boldsymbol{x}}=\mathbf{0}$ holds except for the restriction to the equilibrium point. Therefore asymptotic stability can be concluded.

\section{DISCUSSION OF THE CONDITIONS ON THE POTENTIAL ENERGY}

A short discussion adapted from Albu-Schäffer et al. [2005] of the conditions (7) and (8), which ensure that $\overline{\boldsymbol{q}}(\boldsymbol{\theta})$ is a diffeomorphism, will be given in this section together with some simple examples. Condition (7) ensures that for any constant $\boldsymbol{\theta}$ the system has only one equilibrium, which means that the full system state is statically uniquely determined by $\boldsymbol{\theta}$. Loosely speaking, condition (7) says that "the binding forces should grow faster than the diverging forces between $\boldsymbol{\theta}$ and $\boldsymbol{q}$ ". For the very simple example of a vertical pendulum in the gravity field, connected by a torsional spring $k$ to a fixed position (Fig. 1), condition (7) is always satisfied if $k>m g l$. Indeed, for the extreme case that $k<<m g l$ the system would have many isolated equilibrium points for each $\theta$, some of them even unstable. Condition (8) ensures that, given (7), the mapping $\overline{\boldsymbol{q}}(\boldsymbol{\theta})$ is a diffeomorphism, which means that every desired $\boldsymbol{q}$ can be reached at equilibrium using an appropriate $\boldsymbol{\theta}$. Consider systems of point masses connected by springs. The system in Fig. 2(left) would satisfy condition (8) while system in Fig. 2(right) would not, although it satisfies (7). Indeed, in the latter system $q_{2}$ cannot be controlled independently from $q_{1}$ by the inputs $\theta_{1}$ and $\theta_{2}$ only.

Note that the conditions refer only to the potential energy. There might exist inertial couplings between the states which allow stabilization of the system, as in the example from Fig. 3. The system can be indeed stabilized around the upright position Fantoni et al. [2000], but this cannot be achieved based on shaping of potential energy only. In this case, the conditions (7),(8) are not satisfied.

\section{SOLVING THE ALGEBRAIC EQUATION NUMERICALLY}

The presented approach centrally relies on finding a solution to equation (6). Except for very simple cases, this requires to numerically solve the equation. This is a rather
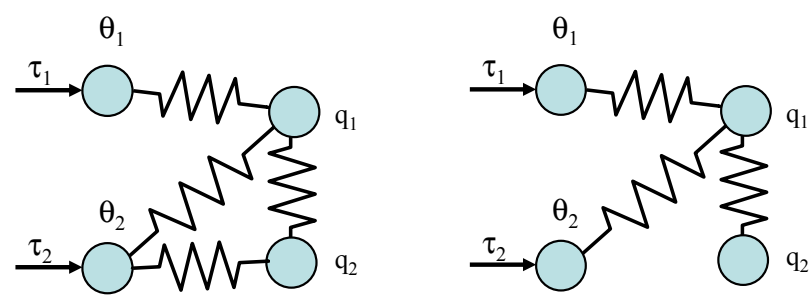

Fig. 2. Left: spring mass system satisfying (7). Right: spring mass system not satisfying (7).

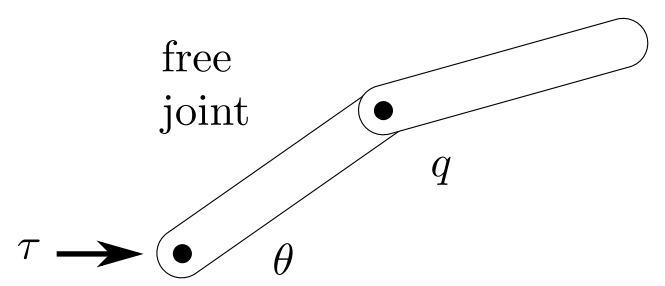

Fig. 3. The acrobot: only one of the two joints is actuated, the second joint can move freely. This underactuated system is not satisfying condition (7)

simple numerical task, since it is ensured that the function $f_{q}$ has only one solution (and its derivative only one minimum). Thus we have a convex optimization problem. Appropriate numerical root finding algorithms will therefore converge from any starting point to the solution. By using a good initial value it will be possible to reach the desired accuracy within very few iteration steps.

Note that equation (6) can be transformed to

$$
\boldsymbol{q}=\boldsymbol{W}(\boldsymbol{\theta}, \boldsymbol{q})
$$

with

$$
\boldsymbol{W}(\boldsymbol{\theta}, \boldsymbol{q})=\boldsymbol{q}-\frac{1}{\alpha_{2}} \frac{\partial U(\boldsymbol{\theta}, \boldsymbol{q})}{\partial \boldsymbol{q}} .
$$

The partial derivative $\frac{\partial \boldsymbol{W}(\boldsymbol{\theta}, \boldsymbol{q})}{\partial \boldsymbol{q}}$ satisfies then, given (7), the inequality

$$
\left\|\frac{\partial \boldsymbol{W}(\boldsymbol{\theta}, \boldsymbol{q})}{\partial \boldsymbol{q}}\right\|=\left\|\boldsymbol{I}-\frac{1}{\alpha_{2}} \frac{\partial^{2} U(\boldsymbol{\theta}, \boldsymbol{q})}{\partial \boldsymbol{q}^{2}}\right\|<\delta<1
$$

for a suitable $\delta$. It follows that

$$
\left\|\boldsymbol{W}\left(\boldsymbol{\theta}, \boldsymbol{q}_{1}\right)-\boldsymbol{W}\left(\boldsymbol{\theta}, \boldsymbol{q}_{2}\right)\right\|<\delta\left\|\boldsymbol{q}_{1}-\boldsymbol{q}_{2}\right\|
$$

and therefore that $\boldsymbol{W}(\boldsymbol{\theta}, \boldsymbol{q})$ is a contraction ${ }^{7}$. The uniqueness of the solution consequently results also from the fixed-point theorem. A way of finding $\overline{\boldsymbol{q}}(\boldsymbol{\theta})$ is to use the fixed-point iteration

$$
\boldsymbol{q}_{i}=\boldsymbol{W}\left(\boldsymbol{\theta}, \boldsymbol{q}_{i-1}\right), i=1,2, \ldots
$$

The result will linearly converge to $\overline{\boldsymbol{q}}$. Newton methods can be further used in order to increase convergence speed. As initial values a reasonable choice $\boldsymbol{q}_{0}=\boldsymbol{q}_{d}$ or $\boldsymbol{q}_{0}=\boldsymbol{\theta}$ would be.

\section{IMPROVING THE CONTROL PERFORMANCE}

The stability of the system is closely related to the passivity property of the controller and the plant and to

\footnotetext{
7 Note that $\boldsymbol{W}(\boldsymbol{\theta}, \boldsymbol{q})$ can be seen as the derivative of the potential
} $U_{T}(\boldsymbol{\theta}, \boldsymbol{q})=1 / 2 \boldsymbol{q}^{T} \boldsymbol{q}-1 / \alpha_{1} U(\boldsymbol{\theta}, \boldsymbol{q})$ with respect to $\boldsymbol{q}$. 


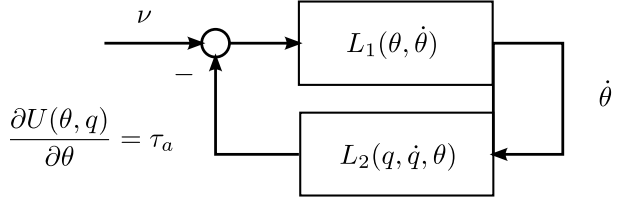

Fig. 4. Passive interconnection of two Lagrangian systems.

their feedback interconnection. This involves the very desired robustness property with respect to plant parameter uncertainty and to unmodeled but passive dynamics. However, it also implies some limitations of the control performance. A reason therefore is the restriction to the feedback of the collocated variables $\boldsymbol{\theta}, \dot{\boldsymbol{\theta}}$.

The controller is based so far on collocated measurements only. In some particular situations, the presented approaches can be extended in order to include also noncollocated feedback for improving the transient performance while remaining within the passivity framework. Since the example and experiments include this noncollocated feedback loop, the approach, first introduced in Albu-Schäffer et al. [2005], is further developed in the following.

Consider therefore in this section the more restricted class of purely elastically coupled underactuated systems, meaning that there is no inertial coupling between collocated and noncollocated states 8 ". Such systems can be represented as the passive feedback interconnection of two Lagrangian subsystems (Fig. 4), with $\boldsymbol{\tau}_{a}=\frac{\partial U(\boldsymbol{\theta}, \boldsymbol{q})}{\partial \boldsymbol{\theta}}$ being the generalized potential force dual to $\dot{\boldsymbol{\theta}}$ and $\boldsymbol{v}$ being the control input. The two subsystems have the Lagrangians $L_{1}$ and $L_{2}$ such that

$$
L(\boldsymbol{x}, \dot{\boldsymbol{x}})=L_{1}(\boldsymbol{\theta}, \dot{\boldsymbol{\theta}})+L_{2}(\boldsymbol{q}, \dot{\boldsymbol{q}}, \boldsymbol{\theta})
$$

is the overall Lagrangian. This case is relevant for the VIA example, as can be seen in Sec. 8. Then the Lagrange equations of the first, collocated subsystem are:

$$
\frac{d}{d t}\left(\frac{\partial L_{1}(\boldsymbol{\theta}, \dot{\boldsymbol{\theta}})}{\partial \dot{\boldsymbol{\theta}}}\right)-\frac{\partial L_{1}(\boldsymbol{\theta}, \dot{\boldsymbol{\theta}})}{\partial \boldsymbol{\theta}}=\boldsymbol{v}-\boldsymbol{\tau}_{a}
$$

Consider a control input of the form

$$
\boldsymbol{v}=\left(\boldsymbol{I}-\boldsymbol{K}_{t}\right) \boldsymbol{\tau}_{a}+\boldsymbol{K}_{t} \boldsymbol{u}
$$

with $\boldsymbol{K}_{t}$ being a constant positive definite matrix and $\boldsymbol{u}$ a new control input. Equation (50) becomes

$$
\boldsymbol{K}_{t}^{-1}\left[\frac{d}{d t}\left(\frac{\partial L_{1}(\boldsymbol{\theta}, \dot{\boldsymbol{\theta}})}{\partial \dot{\boldsymbol{\theta}}}\right)-\frac{\partial L_{1}(\boldsymbol{\theta}, \dot{\boldsymbol{\theta}})}{\partial \boldsymbol{\theta}}\right]=\boldsymbol{u}-\boldsymbol{\tau}_{a}
$$

If the left hand side of the equation corresponds again to a passive system, now w.r.t. $\left\{\boldsymbol{u}-\boldsymbol{\tau}_{a}, \dot{\boldsymbol{\theta}}\right\}$ and with a new Lagrangian $\hat{L}_{1}(\boldsymbol{\theta}, \dot{\boldsymbol{\theta}})$, then the controller design and the analysis can be continued for the new system with the scaled Lagrangian and the new control input $\boldsymbol{u}$ using the methods from Sec. 4. Note that the controller (51) is not passive but the controlled system allows again a passive representation.

Remark 3: The requirement, that the left hand side (52) is again a Lagrangian systems is usually not satisfied for

\footnotetext{
8 while there might be inertial coupling within each subgroup.
}

arbitrary positive definite $\boldsymbol{K}_{t}$. This is however always true at least for $\boldsymbol{K}_{t}=\gamma I, \gamma>0$. For the example in the next section, in which the inertia matrix of $L_{1}$ is diagonal, $\boldsymbol{K}_{t}$ can also be any diagonal p.d. matrix. General p.d. $\boldsymbol{K}_{t}$ matrices can be used only in very specific cases.

\section{EXAMPLE: VARIABLE STIFFNESS ROBOT}

The control of a VSA robot is presented as an example for the design approach. For covering exactly the large variety of VIA designs existing so far one needs a model as general as the one introduces in Albu-Schäffer et al. [2010]. For simplicity, in this example the following reduced model is assumed:

$$
\begin{aligned}
\boldsymbol{M}(\boldsymbol{q}) \ddot{\boldsymbol{q}}+\boldsymbol{C}(\boldsymbol{q}, \dot{\boldsymbol{q}}) \dot{\boldsymbol{q}}+\boldsymbol{g}(\boldsymbol{q}) & =\boldsymbol{\tau} \\
\boldsymbol{B} \ddot{\boldsymbol{\theta}}+\boldsymbol{\tau} & =\boldsymbol{\tau}_{m} \\
\boldsymbol{\tau} & =\boldsymbol{\psi}(\boldsymbol{\theta}-\boldsymbol{q}, \boldsymbol{\sigma})
\end{aligned}
$$

The vectors $\boldsymbol{q} \in \mathbb{R}^{k}$ and $\boldsymbol{\theta} \in \mathbb{R}^{k}$ contain the link and motor side positions respectively. $\boldsymbol{M}(\boldsymbol{q}) \in \mathbb{R}^{k \times k}, \boldsymbol{C}(\boldsymbol{q}, \dot{\boldsymbol{q}}) \dot{\boldsymbol{q}}$, and $\boldsymbol{g}(\boldsymbol{q}) \in \mathbb{R}^{k}$ are the components of the rigid body dynamics: inertia matrix, centripetal and Coriolis vector, and gravity vector. The vector $\boldsymbol{\tau} \in \mathbb{R}^{k}$ represents the joint torques, $\boldsymbol{\tau}_{\text {ext }} \in \mathbb{R}^{k}$ the external torques acting on the robot, and $\boldsymbol{\tau}_{m} \in \mathbb{R}^{k}$ the motor torques. $\boldsymbol{B}=\operatorname{diag}\left(B_{i}\right) \in \mathbb{R}^{k \times k}$ is the diagonal, positive definite motor inertia matrix. The vector $\boldsymbol{\psi}(\boldsymbol{\theta}-\boldsymbol{q}, \boldsymbol{\sigma})$ describes the nonlinear torque characteristics of the VSA joints, see Fig. 5. Each element belongs to a

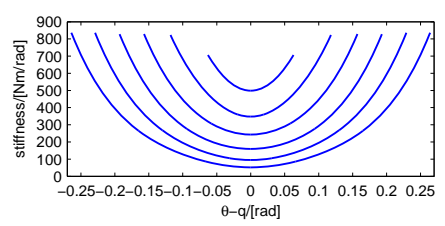

Fig. 5. The stiffness characteristic of the variable stiffness actuators as implemented in the DLR Hand Arm system. The curve family represents increasing stiffness adjustment parameters $\boldsymbol{\sigma}=0-0.175 \mathrm{rad}$. The stiffness curves are symmetric around the equilibrium position.

strictly increasing function family, parameterized by the position $\boldsymbol{\sigma}$ of a second, stiffness adjusting actuator. In this example the dynamics of this actuator is ignored, being in general faster than the main actuator dynamics. Considering this dynamics as well corresponds to the general case stated in Sec. 2 and will be subject of an extended version of the paper. $\boldsymbol{\psi}(\boldsymbol{\theta}-\boldsymbol{q}, \boldsymbol{\sigma})$ is obtained as the derivative of an elastic potential $V_{\psi}(\boldsymbol{q}-\boldsymbol{\theta}, \boldsymbol{\sigma})$ which satisfies properties (7), (8). The conditions imply that the instantaneous stiffness of the VS actuators is upper and lower bounded, which is always the case for a real mechanical system.

\subsection{Torque feedback: shaping the kinetic energy}

Notice that subsystem (54) has the structure from (50), with

$$
L_{1}=\frac{1}{2} \dot{\boldsymbol{\theta}}^{T} \boldsymbol{B} \dot{\boldsymbol{\theta}}, \quad \boldsymbol{\tau}_{a} \hat{=} \boldsymbol{\tau} \quad \boldsymbol{x}_{1} \hat{=} \boldsymbol{\theta}, \quad \boldsymbol{v} \hat{=} \boldsymbol{\tau}_{m}
$$

A torque feedback of the form 


$$
\boldsymbol{\tau}_{m}=\boldsymbol{B} \boldsymbol{B}_{\theta}^{-1} \boldsymbol{u}+\left(\boldsymbol{I}-\boldsymbol{B} \boldsymbol{B}_{\theta}^{-1}\right) \boldsymbol{\tau}
$$

leads to a new subsystem with scaled motor inertia.

$$
\boldsymbol{B}_{\theta} \ddot{\boldsymbol{\theta}}+\boldsymbol{\tau}=\boldsymbol{u} .
$$

The torque controller can therefore be interpreted as a scaling of the kinetic energy of the rotors in order to reduce the vibrations caused by the joint flexibility.

\subsection{Regulation of the desired position: shaping the potential energy}

For the new system described by (53), (57), the controllers developed in Sec. 4 will be applied. The system has the form presented in Sec. 2 with

$$
\begin{aligned}
& T(\boldsymbol{x})=\frac{1}{2} \dot{\boldsymbol{q}}^{T} \boldsymbol{M}(\boldsymbol{q}) \dot{\boldsymbol{q}}+\frac{1}{2} \dot{\boldsymbol{\theta}}^{T} \boldsymbol{B} \dot{\boldsymbol{\theta}} \\
& U(\boldsymbol{x})=V_{\psi}(\boldsymbol{q}-\boldsymbol{\theta}, \boldsymbol{\sigma})+V_{g}(\boldsymbol{q}),
\end{aligned}
$$

where $V_{g}(\boldsymbol{q})$ is a potential function for $\boldsymbol{g}(\boldsymbol{q})$.

The equations (5), (6) of the equilibrium are in this case

$$
\begin{aligned}
& f_{\theta}(\boldsymbol{\theta}, \boldsymbol{q})=-\boldsymbol{\psi}(\boldsymbol{q}-\boldsymbol{\theta}, \boldsymbol{\sigma})=\boldsymbol{u} \\
& \boldsymbol{f}_{\boldsymbol{q}}(\boldsymbol{\theta}, \boldsymbol{q})=\boldsymbol{\psi}(\boldsymbol{q}-\boldsymbol{\theta}, \boldsymbol{\sigma})+\boldsymbol{g}(\boldsymbol{q})=\mathbf{0} .
\end{aligned}
$$

The new control variable $\overline{\boldsymbol{q}}(\boldsymbol{\theta})$ is given by the the solution of (61) for $\boldsymbol{q}$.

Checking the conditions Condition (7) requires that the second order partial derivative

$$
\frac{\partial^{2} U(\boldsymbol{\theta}, \boldsymbol{q})}{\partial \boldsymbol{q}^{2}}=\frac{\partial^{2} U_{\psi}(\boldsymbol{\theta}, \boldsymbol{q})}{\partial \boldsymbol{q}^{2}}+\frac{\partial \boldsymbol{g}(\boldsymbol{q})}{\partial \boldsymbol{q}}
$$

is a p.d., bounded quadratic form. The condition is normally fulfilled for robots with rotational joints ${ }^{9}$. The condition simply states that the instantaneous joint stiffness should be high enough to sustain the robot in the gravity field.

Condition (8) is also satisfied globally, since

$$
\frac{\partial^{2} U(\boldsymbol{\theta}, \boldsymbol{q})}{\partial \boldsymbol{\theta} \partial \boldsymbol{q}}=\frac{\partial^{2} V_{\psi}(\boldsymbol{\theta}, \boldsymbol{q})}{\partial \boldsymbol{\theta} \partial \boldsymbol{q}} .
$$

Consequently, $\overline{\boldsymbol{q}}(\boldsymbol{\theta})$ is a global diffeomorphism.

It follows from Proposition 1 that the controller

$$
\boldsymbol{u}=\boldsymbol{g}(\overline{\boldsymbol{q}})-\boldsymbol{J}_{\bar{q}}^{T}(\boldsymbol{\theta}) \boldsymbol{K}_{p}\left(\overline{\boldsymbol{q}}-\boldsymbol{q}_{d}\right)-\boldsymbol{K}_{d} \dot{\boldsymbol{\theta}}
$$

globally asymptotically stabilizes the desired position $\boldsymbol{q}_{d}$. The relation $\boldsymbol{f}_{\theta}(\boldsymbol{\theta}, \overline{\boldsymbol{q}})=-\boldsymbol{\psi}(\overline{\boldsymbol{q}}-\boldsymbol{\theta}, \boldsymbol{\sigma})=\boldsymbol{g}(\overline{\boldsymbol{q}})$ derived from $(60),(61)$ has been used in order to write the controller in a more intuitive form. The controller therefore simply consists of PD terms and online gravity compensation based on $\overline{\boldsymbol{q}}$ and an inner torque loop.

\section{EXPERIMENTAL VALIDATION ON THE DLR HAND-ARM SYSTEM}

Sec. 4 introduced a controller based on the link side equivalent variable $\overline{\boldsymbol{q}}$. In the following, the advantage of $\overline{\boldsymbol{q}}$ on the stability of the system in comparison to $\boldsymbol{q}$ is validated in experiments. Furthermore, the static equivalency of the

\footnotetext{
9 It is well known that $\partial \boldsymbol{g}(\boldsymbol{q}) / \partial \boldsymbol{q}$ is bounded in this case.
}

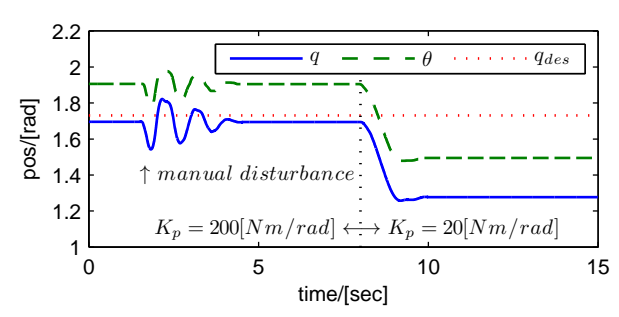

(a) Feedback variable: motor position $\boldsymbol{\theta}$

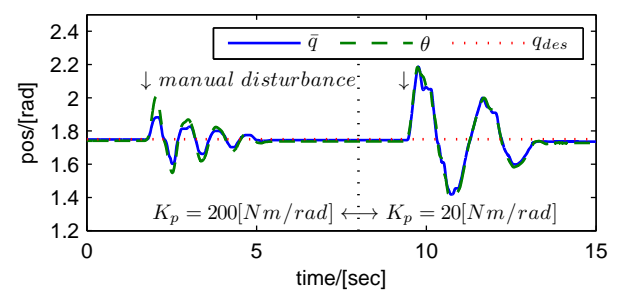

(b) Feedback variable: link side equivalent position $\overline{\boldsymbol{q}}$

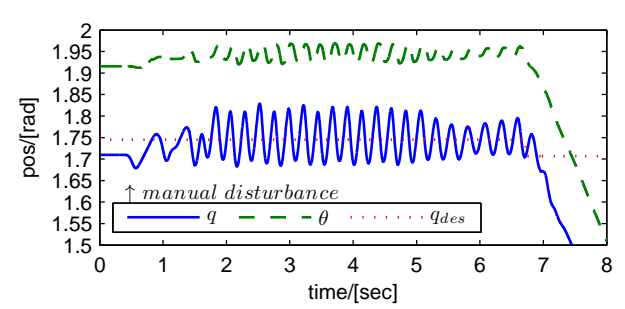

(c) Feedback variable: link side equivalent position $\boldsymbol{q}$

Fig. 6. Measurements of the controllers (65) - (67) on the DLR Hand Arm System. The use of (65) permits to sustain the robot's own weight only for high proportional stiffness gains $K_{P}$, Fig. 6(a). The link side equivalent controller (66) instead shows stable behaviour even for low stiffnesses, see Fig. 6(b). The use of $\boldsymbol{q}$ as feedback variable (67) leads to unstable behaviour, as shown in Fig. 6(c), due to the noncollocation of $\boldsymbol{q}$ and $\boldsymbol{\tau}_{m}$.

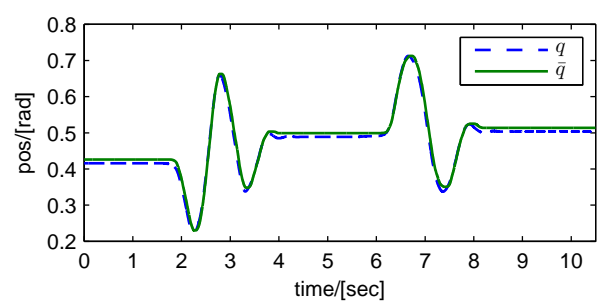

Fig. 7. A measurement of the contraction method to compute $\overline{\boldsymbol{q}}$. The tracking quality of $\boldsymbol{q}$ is very high, only limited by dynamic effect and the model uncertainty.

controller using $\boldsymbol{q}$ and $\overline{\boldsymbol{q}}$ is shown. Finally, the computation procedure based upon contraction mapping as given in Sec. 6 to solve the static equilibrium equation is evaluated.

\subsection{Stability}

To check the stability properties of the proposed approach, the controllers $(10),(27)$ as well as an alternative based on feedback of $\boldsymbol{q}$ were implemented on the VSA robot DLR Hand Arm System Grebenstein et al. [2011], according to 
and

$$
\begin{gathered}
\boldsymbol{u}=\boldsymbol{g}\left(\boldsymbol{q}_{d}\right)-\boldsymbol{K}_{P}\left(\boldsymbol{\theta}-\boldsymbol{\theta}_{d}\right)-\boldsymbol{K}_{D} \dot{\boldsymbol{\theta}} . \\
\boldsymbol{u}=\boldsymbol{g}(\overline{\boldsymbol{q}})-\boldsymbol{J}_{\bar{q}}^{T}(\boldsymbol{\theta}) K_{P}\left(\overline{\boldsymbol{q}}-\boldsymbol{q}_{d}\right)-\boldsymbol{K}_{D} \dot{\boldsymbol{\theta}},
\end{gathered}
$$

$$
\boldsymbol{u}=\boldsymbol{g}(\boldsymbol{q})-\boldsymbol{K}_{P}\left(\boldsymbol{q}-\boldsymbol{q}_{d}\right)-\boldsymbol{K}_{D} \dot{\boldsymbol{\theta}},
$$

The desired torque $\boldsymbol{u}$ is the input command to the torque feedback loop (56). The stability properties of the controllers can be observed in the measurements depicted in Fig. 6. The motor side measurement $\boldsymbol{\theta}$ is used in controller (65) and depicted in Fig. 6(a). The controller (66) makes use of the static equivalent $\overline{\boldsymbol{q}}$ as feedback variable (Fig. 6(b)) and the link side measurement $\boldsymbol{q}$ is used in controller (67) and depicted in Fig. 6(c). A fixed reference position $\boldsymbol{q}_{d}$ is commanded in all three cases. A human disturbed the link impulsively the experiments, at times marked in the plots. No active link side damping control is used, since the elastic property of the spring should not be affected in the considered control mode. The behaviour of the three controllers is:

- Using the motor variable based controller (65) a disturbance results in link oscillations, damped only through the low mechanical joint damping, see (Fig. $6(\mathrm{~b}))$. The link position tracking is good for the static case and high proportional gain $K_{P}=200 \mathrm{Nm} / \mathrm{rad}$ (until $t=8 s$ ). However, for a low gain $K_{P}=$ $20 \mathrm{Nm} / \mathrm{rad}$ (from $t=8 \mathrm{~s}$ ) the robot collapses under its own weight since the lower bound condition on $K_{P}$ is not fulfilled.

- The controller (66) shows similar stable dynamic behaviour as (65) and still provides a statically correct ${ }^{10}$ link side position (Fig. $6(\mathrm{~b})$ ). The advantage of the online gravity compensation based upon $\overline{\boldsymbol{q}}$ can be seen once a low proportional gain $K_{P}$ is commanded, as the robot is able to sustain its own weight and still shows the expected, weakly damped behaviour. This time, the oscillation frequency is lower due to lower overall stiffness, given by the serial interconnection of the physical and the controller spring,

- The behaviour of controller (67) is shown in Fig. $6(\mathrm{c})$. The static link position tracking quality is high. However, after the disturbance is applied, the noncollocated feedback leads to uncontrolled oscillations, until the joint is switched off. The active behaviour of the controller can be observed as the motor motion opposes several times the link motion and thereby injects energy into the system.

\subsection{Contraction Mapping}

The effectiveness of the contraction mapping method (48) is shown in Fig. 7. To generate this plot, the controller (66) with the gains $K_{P}=0$ and $K_{D}=0$ was used, what results in a zero torque controller with gravity compensation. Then the link was disturbed manually. As the motor follows the link motion by the gravity offset, $\overline{\boldsymbol{q}}$ follows the measured $\boldsymbol{q}$ precisely, only limited by dynamic effects and the model uncertainty. The update rate of the controller is $3.33 \mathrm{kHz}$ where 48 is solved each cycle. The constant $\alpha_{2}$ is chosen to be the maximal stiffness value $\alpha_{2}>\boldsymbol{\psi}(\boldsymbol{\theta}-$ $\boldsymbol{q}, \boldsymbol{\sigma})_{\max } \sim 800 \mathrm{Nm} / \mathrm{rad} . \theta_{v}$ is the actual motor position and $\overline{\boldsymbol{q}}_{i-1}$ is the link side equivalent position taken from the last cycle step.

\footnotetext{
${ }^{10}$ Only limited by model uncertainty.
}

A video showing the experiments can be seen on http://www.robotic.dlr.de/336 .

\section{CONCLUSIONS AND OUTLOOK}

The two presented controllers are both well suited for regulating the considered class of underactuated systems based on a robust, collocated, thus passive approach. With the link side equivalent controller, we can exactly reach the desired equilibrium position with arbitrary low feedback gains, thus allowing for very compliant behaviour. The new stability analysis strictly based on energy formulations allows straight-forward treatment of nonlinear stiffness. The approach has been validated on a variable stiffness robot. The presented kinetic energy shaping method can improve considerably the transient performance and provide an almost ideal compliant behaviour. However, additional damping based on the link side velocity is needed in some applications for achieving critical damped behaviour of the system. This issue has been addressed in Petit and Albu-Schäffer [2010] in the context of VIA systems. The integration of that approach into the framework of this paper is subject of current work.

In order to show the uniqueness of the solution $\overline{\boldsymbol{q}}(\boldsymbol{\theta})$ for each $\boldsymbol{\theta}$, let us consider the function $U(\boldsymbol{\theta}, \boldsymbol{q})$ with $\boldsymbol{\theta}=\boldsymbol{\theta}_{0}$ as a parameter $U_{\theta_{0}}=U\left(\boldsymbol{\theta}_{0}, \boldsymbol{q}\right)$. Finding the solutions of

$$
\boldsymbol{f}_{\boldsymbol{q}}\left(\boldsymbol{\theta}_{0}, \boldsymbol{q}\right):=\frac{\partial U_{\boldsymbol{\theta}_{0}}(\boldsymbol{q})}{\partial \boldsymbol{q}}=\mathbf{0}
$$

is equivalent to finding the extremal points of $U_{\boldsymbol{\theta}_{0}}(\boldsymbol{q})$. Since its Hessian $\frac{\partial^{2} U\left(\boldsymbol{\theta}_{0}, \boldsymbol{q}\right)}{\partial \boldsymbol{q}^{2}}$ is positive definite due to $(7)$, it follows that $U_{\boldsymbol{\theta}_{0}}(\boldsymbol{q})$ has one global minimum for each $\boldsymbol{\theta}_{0}$. Thus there is exactly one solution of $\boldsymbol{f}_{\boldsymbol{q}}\left(\boldsymbol{\theta}_{0}, \boldsymbol{q}\right)=0$ and therefore $\overline{\boldsymbol{q}}(\boldsymbol{\theta})$ is a well defined function. Since the norm of the Jacobian of $\overline{\boldsymbol{q}}(\boldsymbol{\theta})$ is lower bounded due to (7), (8)

$$
\left\|\frac{\partial \overline{\boldsymbol{q}}(\boldsymbol{\theta})}{\partial \boldsymbol{\theta}}\right\|>\frac{\alpha_{4}}{\alpha_{2}},
$$

it follows that $\overline{\boldsymbol{q}}(\boldsymbol{\theta})$ is a global diffeomorphism (Zeidler [1986], pp.174).

\section{REFERENCES}

Albu-Schäffer, A., Eiberger, O., Grebenstein, M., Haddadin, S., Ch. Ott, and T. Wimböck, S. Wolf, G.H. (2008). Soft robotics: From torque feedback controlled lightweight robots to intrinsically compliant systems. IEEE Robotics \& Automation Magazine, 20 - 30.

Albu-Schäffer, A. and Hirzinger, G. (2001). A globally stable state-feedback controller for flexible joint robots. Journal of Advanced Robotics, (Special Issue: Selected Papers from IROS 2000), 15(8), 799-814.

Albu-Schäffer, A., Ch. Ott, and Hirzinger, G. (2005). Constructive energy shaping based impedance control for a class of underactuated Euler-Lagrange systems. IEEE Int. Conf. of Robotics and Automation, 13991405.

Albu-Schäffer, A., Ch. Ott, and Hirzinger, G. (2007). A unified passivity based control framework for position, torque and impedance control of flexible joint robots. The International Journal of Robotics Research, 26(1), 23-39. 
Albu-Schäffer, A., Wolf, S., Eiberger, O., Haddadin, S. Petit, F., and Chalon, M. (2010). Dynamic modeling and control of variable stiffness actuators. In IEEE Int. Conf. of Robotics and Automation, 2155-2162.

Bicchi, A. and Tonietti, G. (2004). Fast and Soft Arm Tactics: Dealing with the Safety-Performance Trade-Off in Robot Arms Design and Control. IEEE Robotics and Automation Mag., 11, 22-33.

Blankenstein, G., Ortega, R., and van der Schaft, A.J. (nn). The matching conditions of controlled lagrangians and interconnection and damping assignment passivity based control. Int. Journal of Control, paper from http://www.lss.supelec.fr/perso/ortega/electronic_papers2. 33.

English, C. and Russell, D. (1999). Implementation of variable joint stiffness through antagonistic actuation using rolamite springs. Mechanism and Machine Theory, 34(1), 27-40.

Fantoni, I., Lozano, R., and Spong, M.W. (2000). Energy based control of the pendubot. IEEE Trans. on Automatic Control, 45(4), 725-729.

Grebenstein, M., Albu-Schäffer, A., Bahls, T., Chalone, M., Eiberger, O., Friedl, W., Gruber, R., Hagn, U., Haslinger, R., Höppner, H., Jörg, S., Nickl, M., Nothelfer, A., Petit, F., Reill, J., Seitz, N., Wimböck, T., Wolf, S., Wsthoff, T., and G, H. (2011). Modular statebased behaviour control for safe human-robot interaction: A lightweight control architecture for a lightweight robot. In IEEE Int. Conf. of Robotics and Automation.

Hogan, N. (1985). Impedance control: An approach to manipulation, Part I - Theory, Part II - Implementation, Part III - Applications. ASME J. of Dyn. Systems, Measurement and Control, 107, 1-24.

Hurst, J.W. and Rizzi, A.A. (2008). Series compliance for robot actuation: Application on the electric cable differential leg. IEEE Robotics $\&$ Automation Magazine, $15(3), 2008$.

Koganezawa, K. (2005). Mechanical stiffness control for antagonistically driven joints. In Proc. of the IEEE/RSJ International Conference on Intelligent Robots and Systems, 2512-2519. IEEE/RSJ.

Ch. Ott, Albu-Schäffer, A., Kugi, A., and Hirzinger, G. (2008). On the passivity based impedance control of flexible joint robots. IEEE Transactions on Robotics and Automation, 24(2), 416 - 429.

Migliore, S.A., Brown, E.A., and DeWeerth, S.P. (2005). Biologically Inspired Joint Stiffness Control. In IEEE Int. Conf. on Robotics and Automation (ICRA2005), Barcelona, Spain.

Morita, T., Iwata, H., and Sugano, S. (1999). Development of human symbiotic robot: Wendy. IEEE Int. Conf. of Robotics and Automation, 3183-3188.

Morita, T. and Sugano, S. (1997). Development and evaluation of seven-d.o.f. mia arm. In Proc. 1997 IEEE International Conference on Robotics and Automation, $462-467$.

Ortega, R. and M.Spong (1989). Adaptive motion control of rigid robots: A tutorial. Automatica, 25(10), 877-888.

Ortega, R. and M.Spong (2002). Stabilization of underactuated mechanical systems systems via interconnection and damping assignment. IEEE Trans. on Automatic Control, 47, 1281-1233.
Ortega, R., van der Schaft, A.J., Maschke, B., and Escobar, G. (2002). Interconnection and damping assignment passivity-based control of port-controlled hamiltonian systems. Automatica, paper from http://www.lss.supelec.fr/perso/ortega/electronic_papers2.html, 38(4), nn.

Palli, G., Melchiorri, C., Wimboeck, T., Grebenstein, M., and Hirzinger, G. (2007). Feedback linearization and simultaneous stiffness-position control of robots with antagonistic actuated joints. In IEEE Int. Conf. on Robotics and Automation (ICRA2007), Rome, Italy, 2928-2933.

hilletlit, F. and Albu-Schäffer, A. (2010). State feedback damping control for a multi dof variable stiffness robot arm. IEEE Int. Conf. on Robotics and Automation, submitted.

Petit, F., Chalone, M., Friedl, W., Grebenstein, M., AlbuSchäffer, A., and Hirzinger, G. (2010). Bidirectional antagonistic variable stiffness actuation: Analysis, design and implementation. In IEEE Int. Conf. of Robotics and Automation.

Siciliano, B., Sciavicco, L., and Villani, L. (2009). Robotics: modelling, planning and control. Springer-Verlag.

Takegaki, M. and Arimoto, S. (1981). A new feedback method for dynamic control of manipulators. ASME J. on Dynamic Systems, Measurement and Control, 102, 119-125.

Tomei, P. (1991). A simple PD controller for robots with elastic joints. IEEE Trans. on Automatic Control, 36(10), 1208-1213.

van der Schaft, A. (2002). $L_{2}$ gain and passivity techniques in nonlinear control. Springer-Verlag, second ed. edition.

Vanderborght, B., Verrelst, B., Ham, R.V., Damme, M.V., Lefeber, D., Duran, B.M.Y., and Beyl, P. (2006). Exploiting natural dynamics to reduce energy consumption by controlling the compliance of soft actuators. Int. J. Robotics Research, 25(4), 343-358.

web site, E.P.V. (2011). Variable Impedance Actuators data sheets. In http://www.viactors.org/VSA\%20data\%20sheets.htm.

Wolf, S., Eiberger, O., and Hirzinger, G. (2011). The DLR FSJ: Energy based design of a variable stiffness joint. In ICRA.

Wolf, S. and Hirzinger, G. (2008). A new variable stiffness design: Matching requirements of the next robot generation. In IEEE Int. Conf. on Robotics and Automation, 1741-1746. IEEE, Pasadena, USA.

Zeidler, E. (1986). Nonlinear Functional Analysis and its Applications, volume 1: Fixed-Point Theorems. Springer-Verlag. 